Original paper

\title{
Multi-atlas cardiac PET segmentation
}

\author{
Sally Ji Who Kim ${ }^{\mathrm{a}, \mathrm{b}}$, Seongho Seo ${ }^{\mathrm{a}, \mathrm{c}}$, Hyeon Sik Kim ${ }^{\mathrm{d}}$, Dong-Yeon Kim ${ }^{\mathrm{d}}$, Keon Wook Kanga,e,f, \\ Jung-Joon Min ${ }^{\mathrm{d}}$, Jae Sung Lee $\mathrm{a}^{\mathrm{a}, \mathrm{e}, \mathrm{f}, *}$ \\ ${ }^{a}$ Department of Nuclear Medicine, Seoul National University, Seoul, Republic of Korea \\ ${ }^{\mathrm{b}}$ Gordon Center for Medical Imaging, Department of Radiology, Massachusetts General Hospital, Harvard Medical School, Boston, MA, United States \\ ${ }^{\mathrm{c}}$ Neuroscience Research Institute, Gachon University, Incheon, Republic of Korea \\ ${ }^{\mathrm{d}}$ Department of Nuclear Medicine, Chonnam National University Hwasun Hospital, Hwasun, Jeonnam, Republic of Korea \\ ${ }^{\mathrm{e}}$ Department of Biomedical Sciences, Seoul National University, Seoul, Republic of Korea \\ ${ }_{\mathrm{f}}^{\mathrm{f}}$ Institute of Radiation Medicine, Medical Research Center, Seoul National University, Seoul, Republic of Korea
}

\section{A R T I C L E I N F O}

\section{Keywords:}

Segmentation

Myocardial PET

Atlas

Quantification

\begin{abstract}
A B S T R A C T
Purpose: We propose a multi-atlas based segmentation method for cardiac PET and SPECT images to deal with the high variability of tracer uptake characteristics in myocardium. In addition, we verify its performance by comparing it to the manual segmentation and single-atlas based approach, using dynamic myocardial PET. Methods: Twelve left coronary artery ligated SD rats underwent ( $\left[{ }^{18} \mathrm{~F}\right]$ fluoropentyl) triphenylphosphonium salt PET/CT scans. Atlas-based segmentation is based on the spatial normalized template with pre-defined region-ofinterest (ROI) for each anatomical or functional structure. To generate multiple left ventricular (LV) atlases, each LV image was segmented manually and divided into angular segments. The segmentation methods performances were compared in regional count information using leave-one-out cross-validation. Additionally, the polar-maps of kinetic parameters were estimated.

Results: In all images, the highest $r^{2}$ template yielded the lowest root-mean-square error (RMSE) between the source image and the best-matching templates ranged between 0.91-0.97 and 0.06-0.11, respectively. The single-atlas and multi-atlas based ROIs yielded remarkably different perfusion distributions: only the multi-atlas based segmentation showed equivalent high correlation results $\left(r^{2}=0.92\right)$ with the manual segmentation compared with the single-atlas based $\left(r^{2}=0.88\right)$. The high perfusion value underestimation was remarkable in single-atlas based segmentation.

Conclusions: The main advantage of the proposed multi-atlas based cardiac segmentation method is that it does not require any prior information on the tracer distribution to be incorporated into the image segmentation algorithms. Therefore, the same procedure suggested here is applicable to any other cardiac PET or SPECT imaging agents without modification.
\end{abstract}

\section{Introduction}

Tracer kinetic analysis of dynamic myocardial PET enables the quantification of absolute myocardial blood flow (MBF) and myocardial flow reserve (MFR) in human and animals [1-3]. Dedicated cardiac SPECT systems based on new detector technologies also allow fast dynamic studies and absolute quantitation [4-6]. In the analysis of those myocardial PET and SPECT data, accurate segmentation of left ventricular myocardium is a pivotal step because failure in the myocardial segmentation leads to the wrong quantification of the MBF and MFR $[7,8]$.

Multivariate analysis techniques, such as cluster analysis, factor analysis, independent component analysis, and non-negative matrix factorization, enable the automatic separation of cardiac components and extraction of time-activity curves from dynamic myocardial PET images [9-12]. However, in these approaches, the accuracy of anatomical delineation of the boundary between the myocardium and ventricular cavity is limited, because the cardiac components are separated mainly based on their physiological characteristics. An alternative approach is the deformable segmentation method, such as active contour models and graph cuts [13-17]. Such deformable segmentation methods sometimes fail in the segmentation of myocardium with very low tracer uptake if no appropriate regularization on the contour shape is applied. However, such modeling and regularization parameters

\footnotetext{
* Corresponding author at: Department of Nuclear Medicine, Seoul National University, 103 Daehak-ro, Jongno-gu, Seoul 03080, Republic of Korea.

E-mail address: jaes@snu.ac.kr (J.S. Lee).
} 

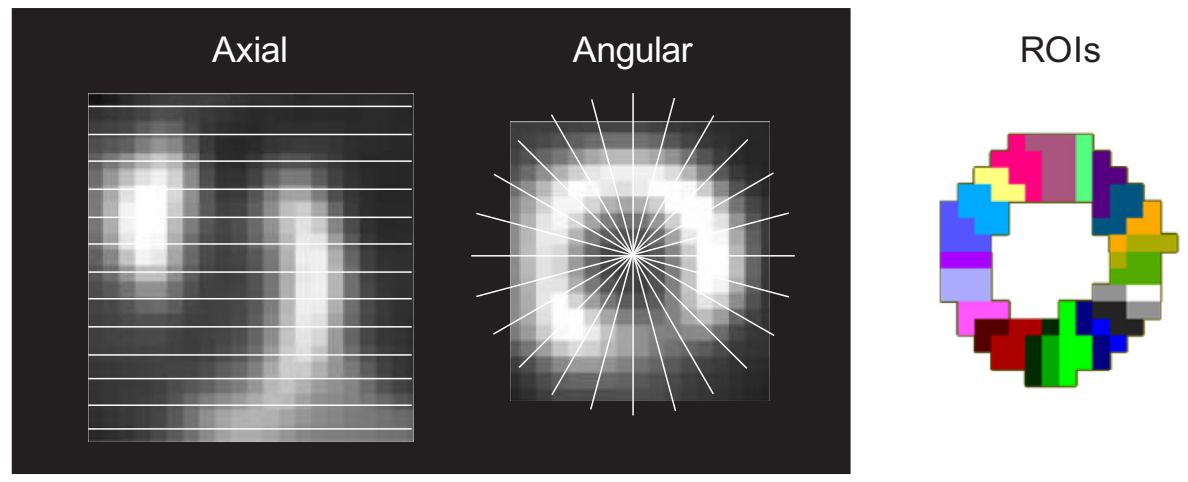

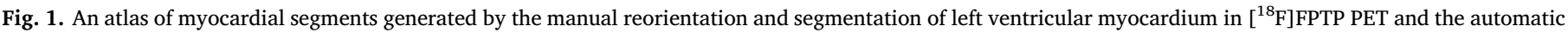
axial and angular $\left(\Delta \theta=15^{\circ}\right)$ subdivision.

optimized for a specific radiotracer and species sometimes do not work for other cardiac imaging agents in the same or other species $[7,8]$. In addition, the activity distribution outside myocardium (e.g., hepatic uptake) that should be properly handled in the segmentation depends on the radiotracers and species. The quantitative gated SPECT (QGS) contour detection principle is a popular tool for gated myocardial SPECT [18]. The modified algorithm, by taking into account the better valve definition of attenuation-corrected high-resolution PET, is also used for the quantitative dynamic PET studies. However, in some cases, user intervention (contour adjustment) is still required [19]. In addition, this approach has mainly been validated only for human data.

In this paper, we propose a multi-atlas based segmentation for cardiac PET and SPECT images to deal with the large variability of the tracer uptake characteristics in the myocardium and other surrounding organs. Atlas-based segmentation is based on the spatial normalization of source (or input) image onto the template on which the regions-ofinterest (ROI) for each anatomical or functional structure is predefined (the atlas labels). Fully automatic segmentation and analysis methods using single or probabilistic atlas-based segmentation are well established for brain PET and SPECT images [20-22]. Commercial software provides the atlas-based segmentation tool for cardiac PET and SPECT image analysis. However, this cardiac segmentation tool relies on only a single standard atlas. Because the spatial normalization between the source image and template is carried out based on the image-based similarity, the large intensity discrepancy between the source and template causes an enormous distortion of the source image. For example, the defect size in the heart is underestimated on the image spatially normalized onto the normal template because the shrinkage of the defect region increases the image similarity. We can overcome this limitation by selecting the best matching template with the most similar tracer distribution as the source image among the multiple template candidates.

In this study, we demonstrate how we have developed the multiatlas based segmentation method for the cardiac image analysis and validated its performance using a fluorine-18 labeled myocardial PET tracer, ( $\left[{ }^{18} \mathrm{~F}\right]$ fluoropentyl)triphenylphosphonium salt $\left(\left[{ }^{18} \mathrm{~F}\right] \mathrm{FPTP}\right)$, as fluorine-18 has better signal to noise ratio than Oxygen-15 and Rubidium-82.

\section{Materials and methods}

\subsection{Data set}

The $\left[{ }^{18} \mathrm{~F}\right]$ FPTP PET dataset acquired in our previous study for the tracer kinetic analysis of this radiotracer was retrospectively used [23], but all PET images were realigned to the one of the images and ROIs were also newly drawn on the realigned PET images. All the preclinical procedures were approved by the Chonnam National University Animal Research Committee and the Guide for the Care and Use of Laboratory
Animals was followed. Twelve Sprague-Dawley rats underwent left coronary artery ligation surgery $24 \mathrm{~h}$ before the PET scan. A small animal PET/CT scanner (Inveon; Siemens Medical Solutions, Knoxville, $\mathrm{TN})$ was used to acquire dynamic PET $(5 \times 1 \mathrm{~s}, 5 \times 5 \mathrm{~s}, 3 \times 10 \mathrm{~s}$, $4 \times 15 \mathrm{~s}, 16 \times 30 \mathrm{~s}, 8 \times 60 \mathrm{~s}: 18 \mathrm{~min}$ ) and static CT images after the intravenous injection of ${ }^{18} \mathrm{~F}$-FPTP (average activity $=37 \mathrm{MBq}$ ). The PET images were reconstructed into $128 \times 128 \times 159$ matrices of $0.78 \times 0.78 \times 0.80-\mathrm{mm}$ voxel size using a filtered back-projection algorithm. A static PET image was generated by summing the dynamic PET frames.

\subsection{Multi-atlas based segmentation}

\subsubsection{Generation of multiple atlases}

The static PET images were cropped to include only the cardiac and surrounding activities and reoriented along the heart axis to form shortaxis slices using FIRE software [24]. The whole left ventricular myocardium in each short-axis slice ( $n=13$ or 14 depending on the animal) was segmented manually and further divided into 24 angular segments $\left(\Delta \theta=15^{\circ}\right)$ as shown in Fig. 1. Accordingly, the total number of myocardial ROIs in each atlas was 312 or 336 . The myocardial ROIs were also merged into 17 segments following the American Heart Association standards. The LV ROIs needed to obtain the arterial input function were drawn on the center of the LV cavity through 14 short-axis slices with the size of $2-10$ voxels per slice to avoid the spill-over contamination from the myocardial activity.

\subsubsection{Atlas-based segmentation}

Fig. 2 shows the schematic diagrams of the single-atlas (a) and multi-atlas (b) based segmentation methods. In the conventional singleatlas based segmentation as shown in Fig. 2a, a template was developed by averaging the static shot-axis images spatially normalized into a single representative image. In the template formation, we used affineonly spatial normalization with 12 transformation parameters (translation, rotation, scaling, and shearing $\times 3$ axes). The ROIs were defined on the single representative image (template) in the above-mentioned way. Thereafter, each source image was spatially normalized into the template using an affine plus a nonlinear transformation. The predefined ROIs were applied to the spatially normalized image to obtain the regional count information. Time-activity curves were obtained by applying the same spatial normalization parameters (affine plus nonlinear) and ROIs to each frame data of the dynamic PET. Performing the inverse transformation of the template and ROIs into the individual image space is an alternative way to reduce the processing time required for resampling all the dynamic frames. However, we did not use this method because of potential interpolation errors that make the inverse transformation and resampling of small ROIs inappropriate.

The core procedure in the multi-atlas based segmentation, shown in Fig. $2 \mathrm{~b}$, is the similarity measurement between the source image and 
a

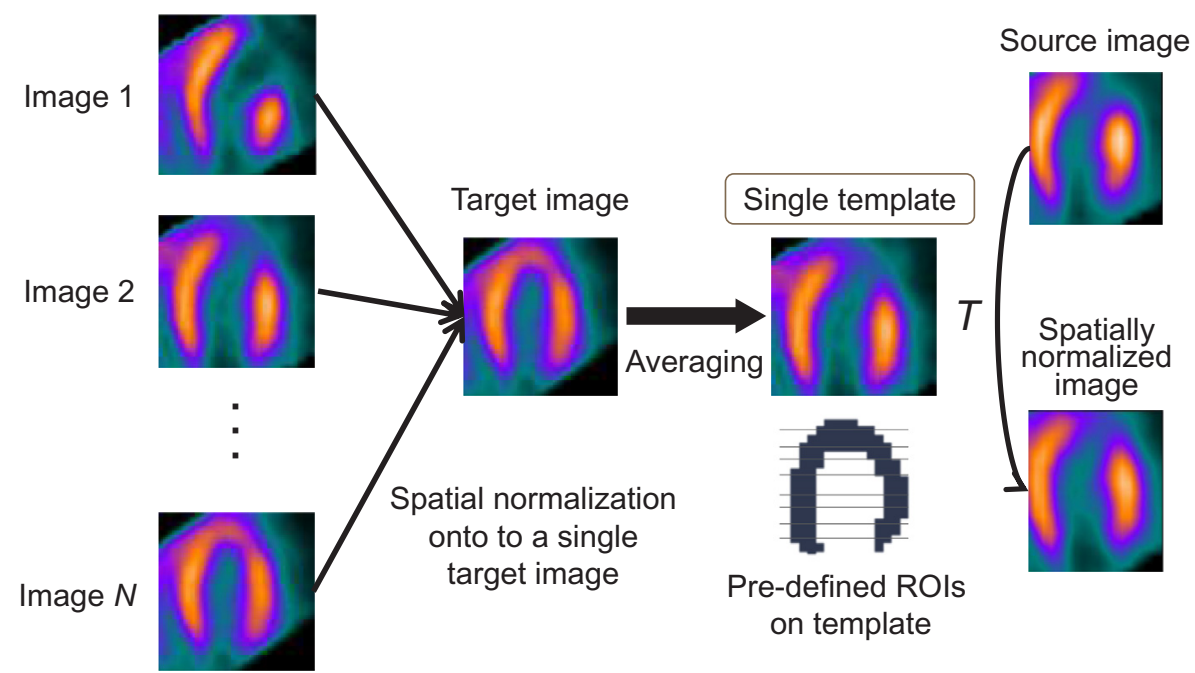

b

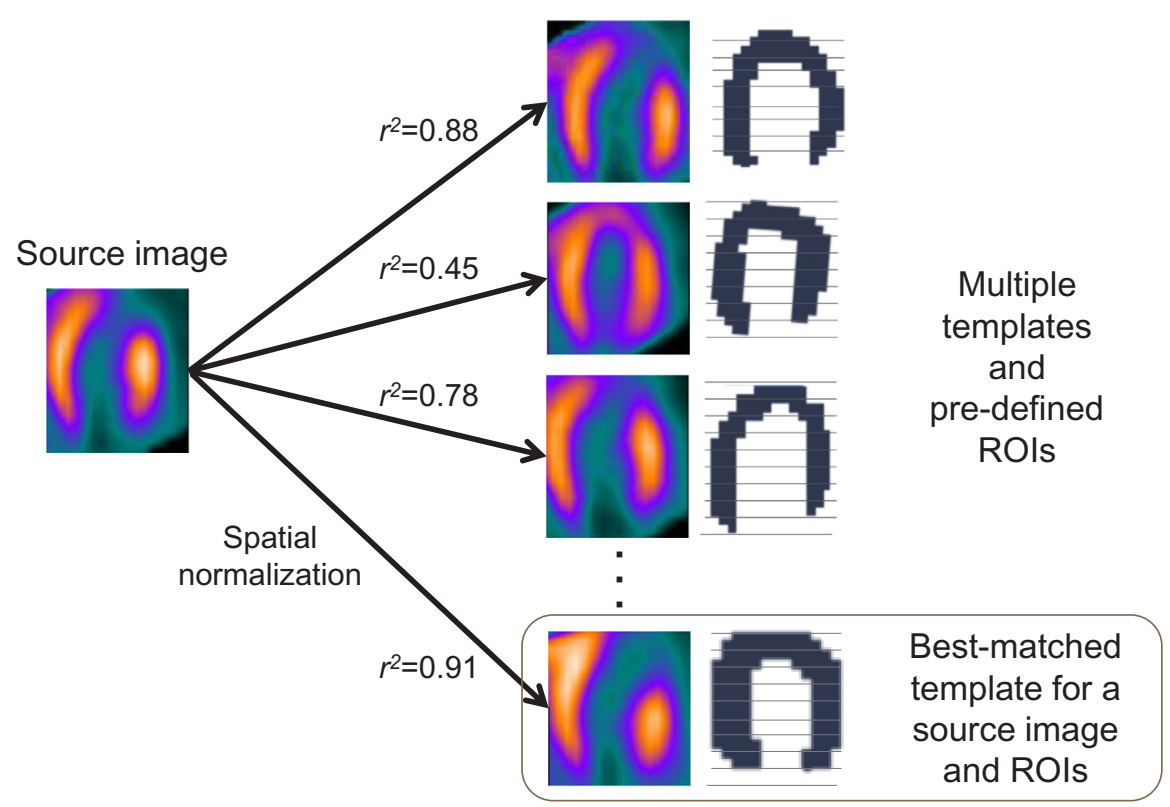

Fig. 2. Schematic diagram of the single-atlas (a) and multi-atlas (b) based segmentation methods.

template. Commonly used similarity measures include the voxel-wise correlation, root-mean square error (RMSE), and mutual information between each template and spatially normalized source image into the template.

There are several different approaches to the multi-atlas based segmentation depending on how one utilizes this similarity measure to select or generate the atlas-based ROIs [25-27]. One of these approaches is the generation of a probabilistic atlas using the similarity measure as a weight optimizer for each source image.

Another way is the selection of the best matched optimal atlas that yields the highest similarity measure. In this study, we adopted the latter approach along with voxel-wise Pearson's correlation and RMSE as the similarity measures. The RMSE was calculated after the count normalization of each image to the average count of highest 30 voxels. The template with the highest correlation and lowest RMSE was selected as the best-matching template. Regional time-activity curves were obtained in the same way as in the single-atlas based segmentation. The Statistical Parametric Mapping (SPM) program (http://fil.ion. ucl.ac.uk) was used for all the spatial normalization procedures.

\subsection{Performance evaluation}

The performance of single-atlas and multi-atlas based segmentation methods were compared using leave-one-out cross-validation. That is, each one of the 12 PET images was analyzed using the single-atlas method, composed of the other 11 images (single-atlas based) or the best-matching atlas, selected from the other 11 images (multi-atlas based). In the single-atlas based approach, the initial image required for the atlas (template and ROIs) formation was randomly selected from the 11 images. In the multi-atlas based approach, the other 11 images, except for the source image itself and ROIs drawn on each of them, were used as the multiple atlases. The similarity of the source image and best-matching image was evaluated with Dice similarity coefficient using the masked images obtained by applying the threshold of $70 \%$ of maximum counts in each image.

For qualitative and quantitative comparisons, we generated polar maps of kinetic parameters. These were estimated using compartmental modeling applied to the dynamic PET images. The parametric images of kinetic parameters $\left(K_{1}\right.$ : uptake, $k_{2}$ : clearance, $V_{\mathrm{a}}$ : blood volume 
a

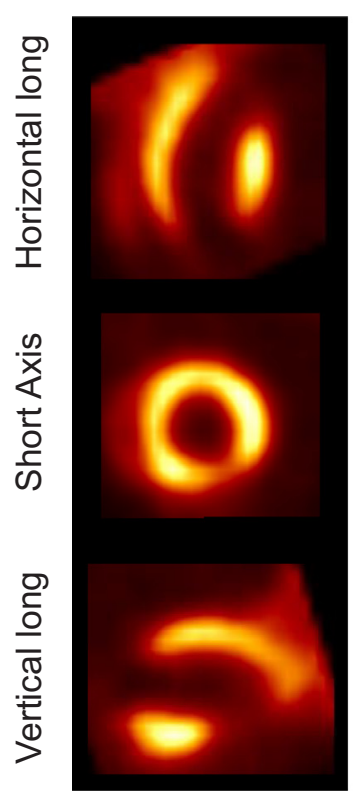

b

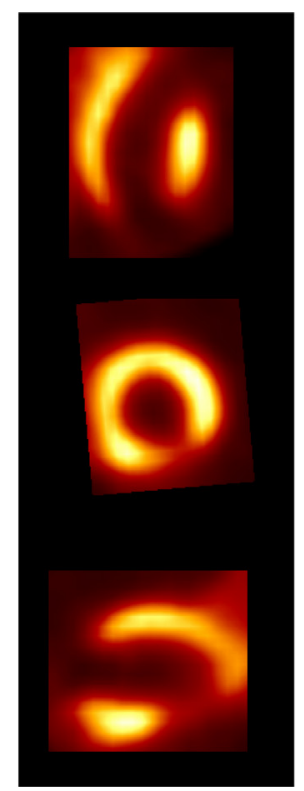

C

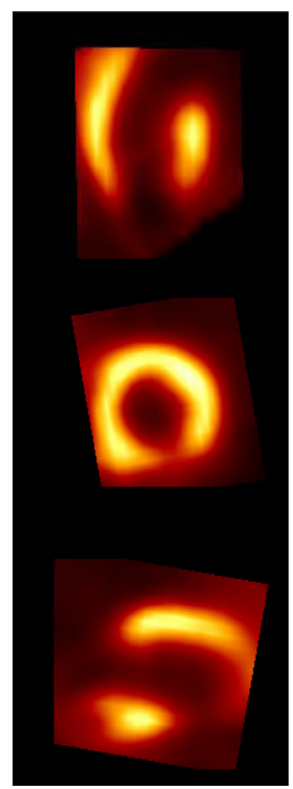

d

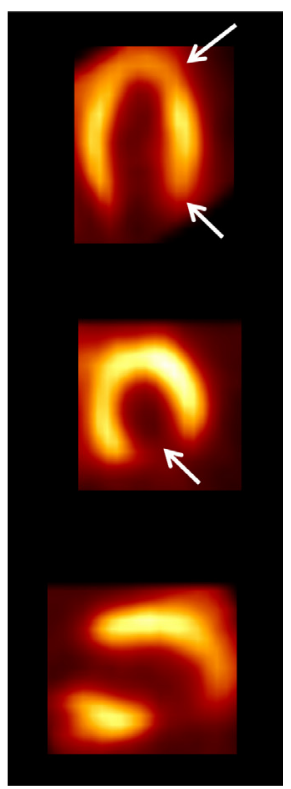

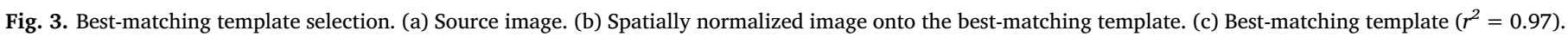
(d) Worst-matching template $\left(r^{2}=0.63\right.$; arrows indicate the unmatched regions between source and template images).

fraction) were produced using two-compartment models and the basis function method as described in [23]. Thereafter, the polar maps of perfusion, $\alpha \cdot K_{1}\left(\alpha=1-V_{\mathrm{a}}\right)$ [28], were developed by applying 1$)$ the manual ROIs drawn on each image, 2) single-atlas based ROIs, and 3) multi-atlas based ROIs to the parametric images. The correlation between the kinetic parameters obtained using the atlas-based methods and manual ROIs were assessed using Pearson's correlation.

\section{Results}

\subsection{Template selection}

Fig. 3 shows the result of the best-matching template selection in the multi-atlas based cardiac segmentation for a representative $\left[{ }^{18} \mathrm{~F}\right] \mathrm{FPTP}$ rat PET data (source image) in which the severe perfusion defect is shown in the anterolateral wall of myocardium. The best-matching template as shown in Fig. 3c, yielding the highest voxel-wise correlation $\left(r^{2}=0.97\right)$ and lowest RMSE (0.0063) with the spatially normalized image as shown in Fig. 3b, exhibits very similar tracer distribution with the source image as shown in Fig. 3a in terms of the location and intensity of the lesion. For comparison, the worst-matching template with the lowest correlation $\left(r^{2}=0.63\right)$ is shown in Fig. 3d. There was a remarkable difference between the worst-matching template and the source image. The results of the voxel-wise comparison between the source image and the other 11 template candidates are presented in Fig. 4.

The range of square of correlation coefficient $\left(r^{2}\right)$, the RMSE and the best- and worst-matching templates for each source image are summarized in Table 1. In all the images, the template with highest $r^{2}$ yielded the lowest RMSE. The $r^{2}$ and RMSE between the source image and the best-matching templates ranged $0.91-0.97$ and $0.06-0.11$, respectively. Each template exhibited different values of $r^{2}$ and RMSE from those of the source images as shown in Table 2.

The dice similarity coefficient between the source image and bestmatching image was $0.87 \pm 0.03$.

The time-activity curves obtained using the manually drawn ROIs and the atlas-based segmentation with the best-matching template agreed well with each other as shown in Fig. 5.

\subsection{Comparison between Single-atlas and multi-atlas based approaches}

Fig. 6 shows the polar maps of myocardial perfusion generated in a representative case using manually drawn ROIs (a), multi-atlas based ROIs (b), and single-atlas based ROIs (c). The single-atlas and multiatlas based ROIs yielded remarkably different perfusion distributions: only the multi-atlas based segmentation exhibited equivalent results with the manual segmentation as shown in Fig. 6.

Fig. 7 shows the linear regression results and Bland-Altman plots for the multi-atlas based versus manually-drawn ROIs (a) and single-atlas based versus manual ROIs (b). All the segmental perfusion values ( $n=204$ ) obtained from the 12 rats were included in the analysis. The multi-atlas based segmentation method yielded a higher correlation with the manual segmentation method $\left(r^{2}=0.92\right)$ than the correlation obtained by the single-atlas based one with the manual segmentation method $\left(r^{2}=0.88\right)$. The underestimation of perfusion obtained by applying the single-atlas based segmentation was remarkable in the ROIs with high perfusion values.

\section{Discussion}

The atlas-based segmentation is a robust method that has been mainly used for brain image analysis in nuclear medicine. The singleatlas based one is computationally efficient because it requires only the single execution of spatial normalization [29]. However, the accuracy of this approach is limited because one cannot perfectly deal with the change of morphology and tracer distribution in severe pathological conditions with the single atlas or template. The multi-atlas based segmentation involves a greater computational burden than the singleatlas based one; however, the enhanced accuracy in the assessment of tracer uptake and quantitative parameters, as evidenced in this study, can justify its use for nuclear medicine image analysis [25-27]. In addition, cost effective parallel computation tools are widely available now.

In this study, we suggested the multi-atlas based segmentation for cardiac PET images and verified its performance by comparing it to the manual segmentation. The multi-atlas based segmentation outperformed the conventional single-atlas based one in comparison to manual segmentation. Although only the cardiac PET images of 

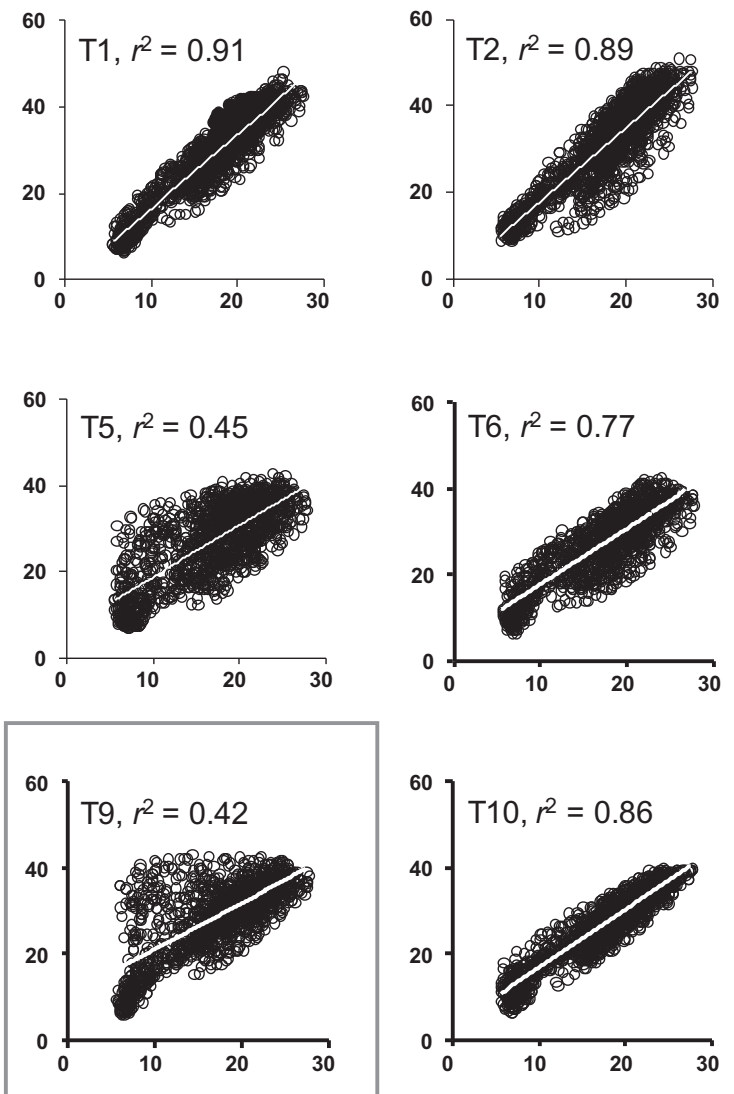

Worst template
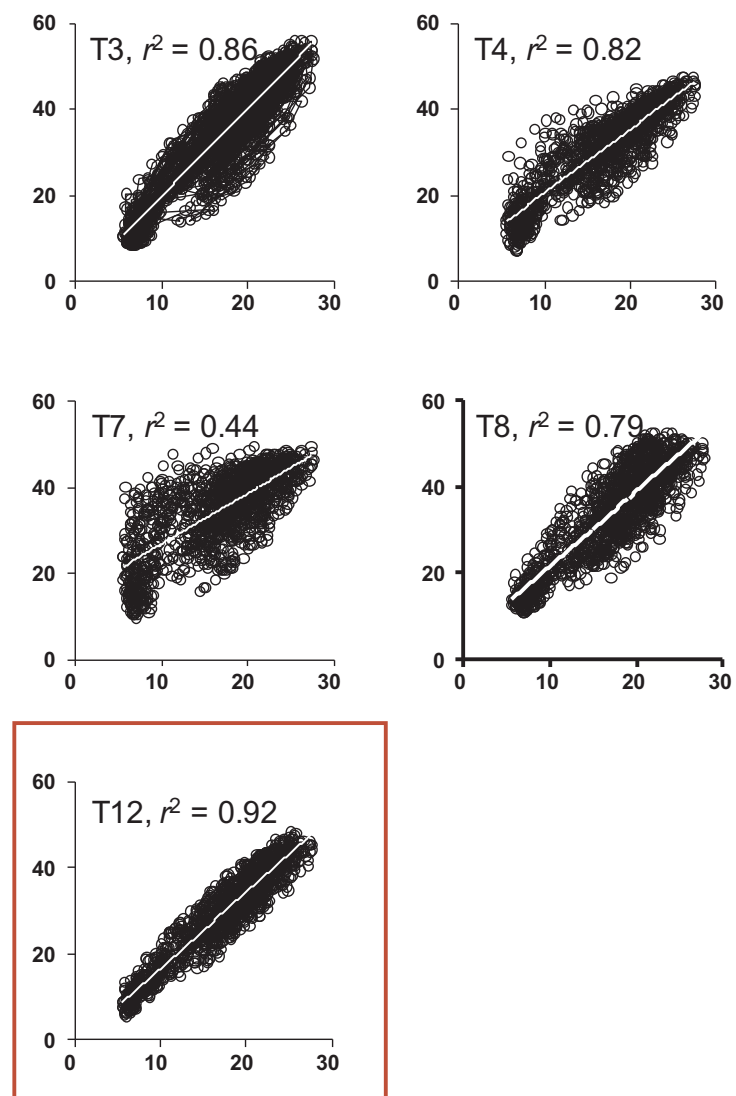

Best template

Fig. 4. Voxel-wise comparison between a source image (image 11) and the other 11 template candidates (templates 1-10 and 12).

Table 1

The range of square of correlation coefficient $\left(r^{2}\right)$ and root-mean-square error (RMSE) of normalized count for each source image.

\begin{tabular}{lll}
\hline Source Image (\#) & $r^{2}$ & RMSE \\
\hline 1 & $0.67-0.96$ & $0.06-0.15$ \\
2 & $0.47-0.97$ & $0.06-0.26$ \\
3 & $0.63-0.97$ & $0.06-0.18$ \\
4 & $0.73-0.95$ & $0.07-0.15$ \\
5 & $0.57-0.93$ & $0.05-0.22$ \\
6 & $0.67-0.96$ & $0.07-0.16$ \\
7 & $0.55-0.95$ & $0.06-0.22$ \\
8 & $0.42-0.94$ & $0.09-0.21$ \\
9 & $0.51-0.91$ & $0.11-0.23$ \\
10 & $0.65-0.95$ & $0.07-0.16$ \\
11 & $0.42-0.92$ & $0.10-0.25$ \\
12 & $0.69-0.95$ & $0.08-0.15$ \\
\hline Average & $0.58-0.95$ & $0.07-0.20$ \\
\hline
\end{tabular}

myocardial infarction model induced by the left coronary artery ligation were employed in this study, the voxel-wise correlation between a single template and the other spatially normalized images onto the template was highly variable as shown in Table 2. Consequently, the single-atlas based segmentation yielded a larger discrepancy relative to the manual segmentation in the quantification of the perfusion parameter as shown in Fig. 7b. On the contrary, the best-matching templates in the multi-atlas based segmentation exhibited over 0.90 voxel-wise correlation for all source images. This high correlation between the template and source images in each individual data analysis led to the excellent agreement level in the segmental analysis of perfusion parameter as shown in Figs. 6 and 7. The close proximity between source image and best matched template would be useful for minimizing the
Table 2

The range of square of correlation coefficient $\left(r^{2}\right)$ and root-mean-square error (RMSE) of normalized count for each template.

\begin{tabular}{lll}
\hline Template (\#) & $r^{2}$ & RMSE \\
\hline 1 & $0.91-0.97$ & $0.06-0.12$ \\
2 & $0.75-0.96$ & $0.08-0.20$ \\
3 & $0.71-0.96$ & $0.06-0.17$ \\
4 & $0.77-0.91$ & $0.07-0.13$ \\
5 & $0.45-0.89$ & $0.06-0.23$ \\
6 & $0.77-0.92$ & $0.07-0.14$ \\
7 & $0.42-0.88$ & $0.05-0.21$ \\
8 & $0.79-0.91$ & $0.10-0.16$ \\
9 & $0.42-0.81$ & $0.12-0.26$ \\
10 & $0.74-0.92$ & $0.07-0.13$ \\
11 & $0.51-0.91$ & $0.11-0.23$ \\
12 & $0.89-0.97$ & $0.06-0.11$ \\
\hline Average & $0.68-0.92$ & $0.08-0.17$ \\
\hline
\end{tabular}

error in the registration of soft tissue that is considered extremely difficult $[30,31]$.

The main advantage of the proposed multi-atlas based cardiac segmentation method is that it does not require any prior information on the tracer distribution to be incorporated into the image segmentation algorithms. In this proposed method, no adjustment of any threshold or regularization parameters is necessary. Therefore, the same procedure, as shown in Fig. $2 \mathrm{~b}$, can be applied to any other routinely used or investigative cardiac PET or SPECT imaging agents without modification. In addition, there is no need to provide initial seed pixel or ROI for contour evolving [16]. However, the accurate segmentation of myocardium to compose the multiple atlases is a prerequisite step demanding manual or semi-automatic delineation of 

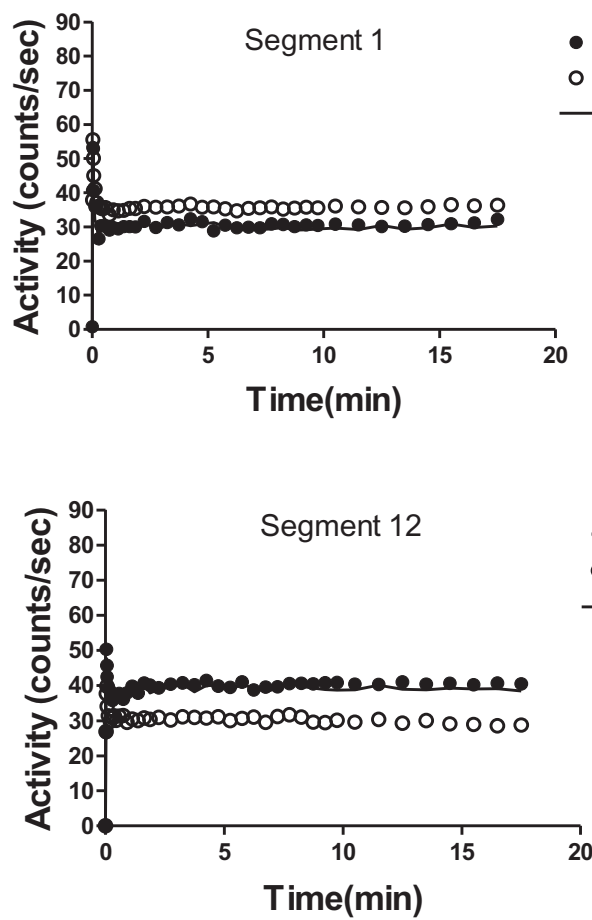
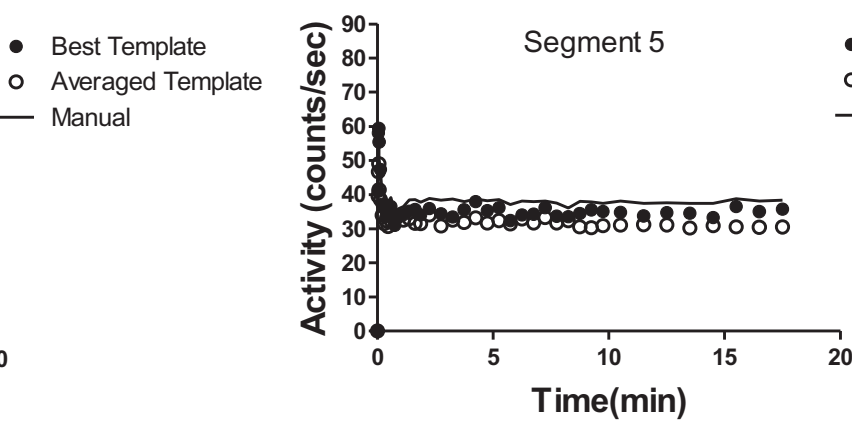

- Best Template

- Averaged Template

- Manual

Fig. 5. Time-activity curves obtained using the multi-atlas

(a) and single-atlas (O) based segmentation. The solid line was obtained using the manual ROIs.

a

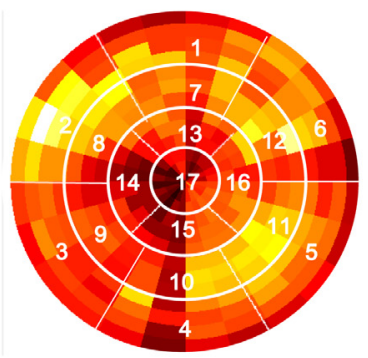

b

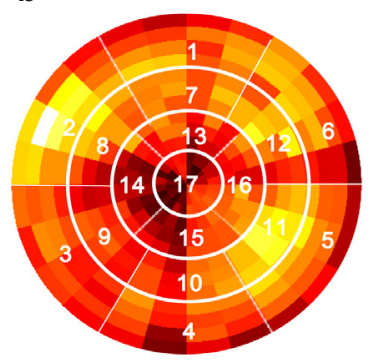

C

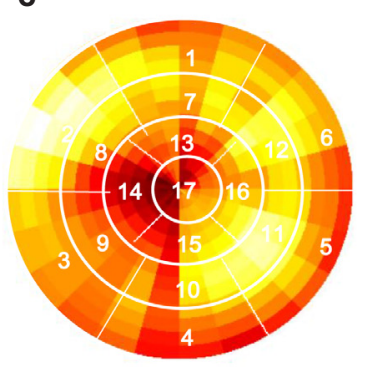

- Best Template

- Averaged Template

- Manual

Fig. 6. Polar maps of myocardial perfusion generated in a representative case using manually drawn (a), multi-atlas based (b), and single-atlas based (c) ROIs.

endocardial and epicardial boundaries of the left ventricular myocardium. The necessary number of atlases for sufficiently good segmentation results would depend on the variability of image patterns in normal and pathological conditions. Further investigation is warranted to determine the required number of atlases for more diverse cases of cardiovascular diseases. It should be also investigated whether different reconstruction algorithms (for example, the most recent iterative reconstruction algorithms with resolution modeling in respect to FBP) or cardiac motion (gated images instead of summed ones) influence the result of the study. Because the advanced reconstruction algorithms and cardiac gated images yield better contrast between the myocardium and blood pool and thinner thickness of myocardium, different reconstruction algorithms or cardiac motion management would lead to decreased correlation between the template and individual source image. Although it is likely that same best matched optimal atlas will be selected by the similarity measure used in this study, the performance of spatial resolution would be more influenced by the reconstruction algorithms and cardiac motion management. Whether denoising images will improve our template-based segmentation is an interesting future research topic [32]. In addition, segmenting myocardium in PET using fast-growing deep learning approach that outperforms conventional signal and image processing algorithms for some applications is of interest [33-37]. Also, the generation of synthetic lesions in PET images will be a useful method to compare the performance of different approaches for myocardial segmentation [32,38,39].

\section{Conclusions}

We developed a multi-atlas based cardiac segmentation method for a fluorine-18 labeled cardiac PET imaging agent and demonstrated its feasibility by comparing the quantitative perfusion parameter values obtained using the proposed method with those obtained by manual segmentation. The similarity between the template and spatially normalized source image was significantly higher in the multi-atlas based approach than in the single-atlas based approach.

\section{Acknowledgments}

This work was supported by grants from the Korea Healthcare Technology R\&D Project, Ministry of Health \& Welfare, Republic of Korea (HI13C0163020015) and the National Research Foundation of Korea (NRF) funded by the Korean Ministry of Science and ICT (grant no. NRF-2014M3C7034000 and NRF-2016R1A2B3014645). The funding source had no involvement in the study design, collection, analysis, or interpretation. 
a

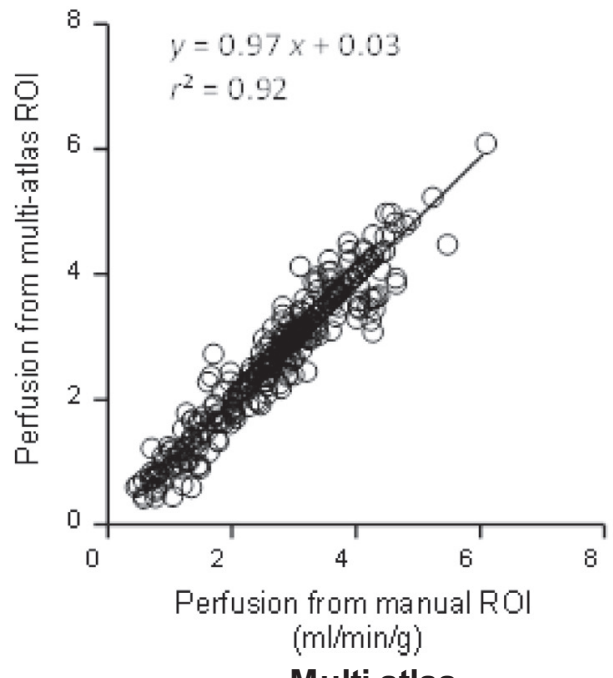

Multi atlas

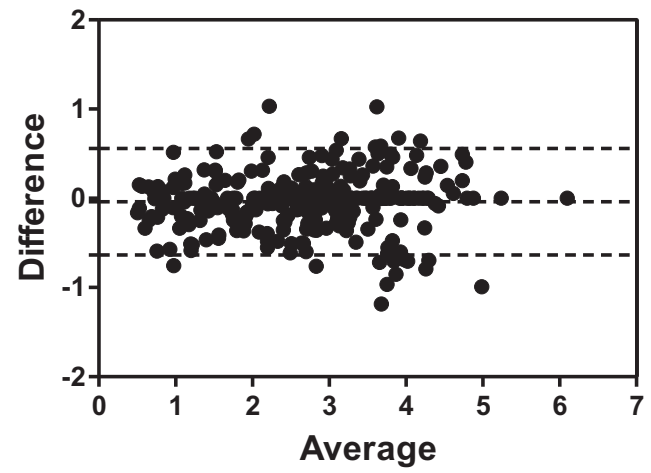

b

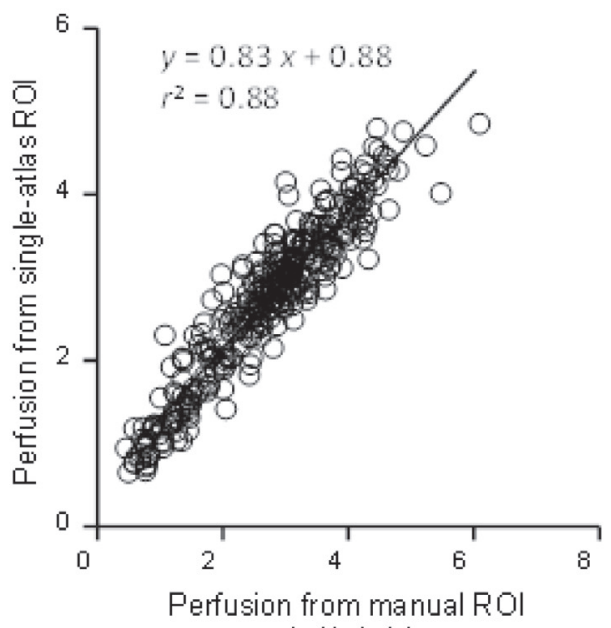

$(\mathrm{m} / \mathrm{min} / \mathrm{g})$

Single atlas

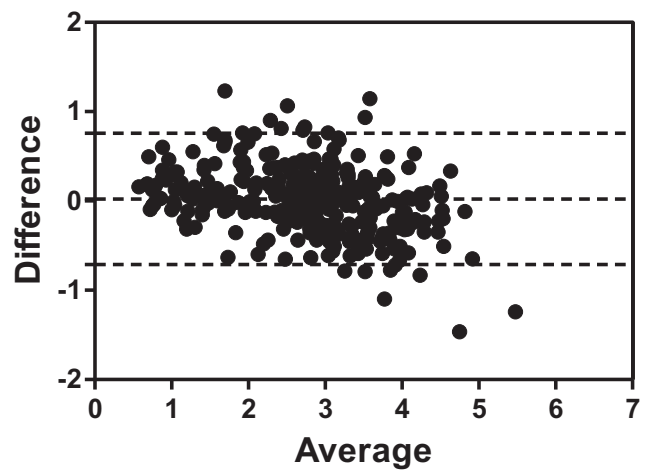

Fig. 7. Linear regression results and Bland-Altman plots for multi-atlas based versus manual ROIs (a) and single-atlas based versus manual ROIs (b).

\section{Compliance with ethical standards}

Conflict of interest: The authors declare that they have no conflict of interest.

\section{References}

[1] Iida H, Kanno I, Takahashi A, Miura S, Murakami M, Takahashi K, et al. Measurement of absolute myocardial blood flow with $\mathrm{H}_{2}{ }^{15} \mathrm{O}$ and dynamic positronemission tomography. Strategy for quantification in relation to the partial-volume effect. Circulation 1988;78:104-15.

[2] Lee JS, Lee DS, Ahn JY, Yeo JS, Cheon GJ, Kim SK, et al. Generation of parametric image of regional myocardial blood flow using $\mathrm{H}_{2}{ }^{15} \mathrm{O}$ dynamic PET and a linear least-squares method. J Nucl Med 2005;46:1687-95.

[3] Sherif HM, Nekolla SG, Saraste A, Saraste A, Reder S, Yu M, et al. Simplified quantification of myocardial flow reserve with flurpiridaz F 18: validation with microspheres in a pig model. J Nucl Med 2011;52:617-24.

[4] Wells RG, Timmins R, Klein R, Lockwood J, Marvin B, deKemp RA, et al. Dynamic SPECT measurement of absolute myocardial blood flow in a porcine model. J Nucl Med 2014;55:1685-91.

[5] Nkoulou R, Fuchs TA, Pazhenkottil AP, Kuest SM, Ghadri JR, Stehli J, et al. Absolute myocardial blood flow and flow reserve assessed by gated SPECT with cadmium-zinc-telluride detectors using ${ }^{99 \mathrm{~m}} \mathrm{Tc}$-Tetrofosmin: head-to-head comparison with ${ }^{13} \mathrm{~N}$-ammonia PET. J Nucl Med 2016;57:1887-92.

[6] Lee JS, Kovalski G, Sharir T, Lee DS. Advances in imaging instrumentation for nuclear cardiology. J Nucl Cardiol 2017. https://doi.org/10.1007/s12350-0170979-8.

[7] Nesterov SV, Deshayes E, Sciagrà R, Settimo L, Declerck JM, Pan XB, et al. Quantification of myocardial blood flow in absolute terms using ${ }^{82} \mathrm{Rb}$ PET imaging: the RUBY-10 study. JACC: Cardiovascular Imaging 2014;7:1119-27.

[8] Dunet V, Klein R, Allenbach G, Renaud J, Prior JO. Myocardial blood flow quantification by Rb-82 cardiac PET/CT: a detailed reproducibility study between two semi-automatic analysis programs. J Nucl Cardiol 2016;23:499-510.

[9] Ahn JY, Lee DS, Lee JS, Kim SK, Cheon GJ, Yeo JS, et al. Quantification of regional myocardial blood flow using dynamic $\mathrm{H}_{2}{ }^{15} \mathrm{O}$ PET and factor analysis. J Nucl Med 2001;42:782-7.

[10] Lee JS, Lee DD, Choi S, Lee DS. Application of nonnegative matrix factorization to dynamic positron emission tomography. In: 3rd Int. Conf. on Independent Component Analysis and Blind Signal Separation 2001. 629-32.

[11] Lee JS, Lee DS, Ahn JY, Cheon GJ, Kim SK, Yeo JS, et al. Blind separation of cardiac components and extraction of input function from $\mathrm{H}_{2}{ }^{15} \mathrm{O}$ dynamic myocardial PET using independent component analysis. J Nucl Med 2001;42:938-43.

[12] Harms HJ, Knaapen P, de Haan S, Halbmeijer R, Lammertsma AA, Lubberink M. Automatic generation of absolute myocardial blood flow images using $\left[{ }^{15} \mathrm{O}_{\mathrm{H}} \mathrm{O}\right.$ and a clinical PET/CT scanner. Eur J Nucl Med Mol Imaging 2011;38:930-9.

[13] Pluempitiwiriyawej C, Moura JM, Wu YJ, Ho C. STACS: New active contour scheme for cardiac MR image segmentation. IEEE Trans. Med. Imaging 2005;24:593-603.

[14] Cheng-Liao J, Qi J. Segmentation of mouse dynamic PET images using a multiphase level set method. Phys Med Biol 2010;55:6549-69.

[15] Kang HC, Kim B, Lee J, Shin J, Shin Y-G. Automatic left and right heart segmentation using power watershed and active contour model without edge. Biomed Eng Lett 2014:4:355-61.

[16] Abdalbari A, Ren J, Green M. Seeds classification for image segmentation based on 3-D affine moment invariants. Biomed Eng Lett 2016;6:224-33.

[17] Hajiaghayi M, Groves EM, Jafarkhani H, Kheradvar A. A 3-D active contour method for automated segmentation of the left ventricle from magnetic resonance images. IEEE Trans Biomed Eng 2017;64:134-44.

[18] Germano G, Kiat H, Kavanagh PB, Moriel M, Mazzanti M, Su HT, et al. Automatic quantification of ejection fraction from gated myocardial perfusion SPECT. J Nucl Med 1995;36:2138-47.

[19] Slomka PJ, Alexanderson E, Jacome R, Jiménez M, Romero E, Meave A, et al. Comparison of clinical tools for measurements of regional stress and rest myocardial blood flow assessed with ${ }^{13} \mathrm{~N}$-ammonia PET/CT. J Nucl Med 2012;53:171-81.

[20] Kang KW, Lee DS, Cho JH, Lee JS, Yeo JS, Lee SK, et al. Quantification of F-18 FDG PET images in temporal lobe epilepsy patients using probabilistic brain atlas. Neuroimage 2001;14:1-6.

[21] Lee DS, Lee JS, Oh SH, Kim SK, Kim JW, Chung JK, et al. Cross-modal plasticity and cochlear implants. Nature 2001;409:149-50.

[22] Lee SK, Lee DS, Yeo JS, Lee JS, Kim YK, Jang MJ, et al. FDG-PET images quantified by probabilistic atlas of brain and surgical prognosis of temporal lobe epilepsy. 
Epilepsia 2002;43:1032-8.

[23] Kim JW, Seo S, Kim HS, Kim DY, Lee HY, Kang KW, et al. Comparative evaluation of the algorithms for parametric mapping of the novel myocardial PET imaging agent ${ }^{18}$ F-FPTP. Ann Nucl Med 2017;31:469-79.

[24] Lee JS, Park KS, Lee DS, Lee CW, Chung JK, Lee MC. Development and applications of a software for Functional Image Registration (FIRE). Comput. Methods Programs Biomed 2005;78:157-64.

[25] Aljabar P, Heckemann RA, Hammers A, Hajnal JV, Rueckert D. Multi-atlas based segmentation of brain images: atlas selection and its effect on accuracy. Neuroimage 2009;46:726-38.

[26] Lötjönen JM, Wolz R, Koikkalainen JR, Thurfjell L, Waldemar G, Soininen H, et al. Fast and robust multi-atlas segmentation of brain magnetic resonance images. Neuroimage 2010;49:2352-65.

[27] Wang H, Suh JW, Das SR, Pluta JB, Craige C, Yushkevich PA. Multi-atlas segmentation with joint label fusion. IEEE Trans Pattern Anal Machine Intel 2013;35:611-23.

[28] Iida H, Rhodes CG, de Silva R, Yamamoto Y, Araujo LI, Maseri A, et al. Myocardial tissue fraction-correction for partial volume effects and measure of tissue viability. J Nucl Med 1991;32:2169-75.

[29] Lee JS, Lee DS, Kim SK, Lee SK, Chung JK, Lee MC, et al. Localization of epileptogenic zones in F-18 FDG brain PET of patients with temporal lobe epilepsy using artificial neural network. IEEE Trans Med Imaging 2000;19:347-55.

[30] Huang X, Ren J, Abdalbari A, Green M. Vessel-based fast deformable registration with minimal strain energy. Biomed Eng Lett 2016;6:47-55.
[31] Ren J, Green M, Huang X, Abdalbari A. Automatic error correction using adaptive weighting for vessel-based deformable image registration. Biomed Eng Lett 2017;7:173-81.

[32] Huerga C, Glaría L, Castro P, Alejo L, Bayón J, Guibelalde E. Segmentation improvement through denoising of PET images with 3D-context modelling in wavelet domain. Phys Med 2018;53:62-71.

[33] Mansour RF. Deep-learning-based automatic computer-aided diagnosis system for diabetic retinopathy. Biomed Eng Lett 2018;8:41-57.

[34] Dey D, Chaudhuri S, Munshi S. Obstructive sleep apnoea detection using convolutional neural network based deep learning framework. Biomed Eng Lett 2018;8:95-100.

[35] Park J, Hwang D, Kim KY, Kang SK, Kim YK, Lee JS. Computed tomography superresolution using deep convolutional neural network. Phys Med Biol 2018;63:145011.

[36] Hwang D, Kim KY, Kang SK, Seo S, Paeng JC, Lee DS, et al. Improving the accuracy of simultaneously reconstructed activity and attenuation maps using deep learning. J Nucl Med 2018;59:1624-9.

[37] Kang SK, Seo S, Shin SA, Byun MS, Lee DY, Kim YK, et al. Adaptive template generation for amyloid PET using a deep learning approach. Hum Brain Mapp 2018.

[38] Berthon B, Häggström I, Apte A, Beattie BJ, Kirov AS, Humm JL, et al. PETSTEP: Generation of synthetic PET lesions for fast evaluation of segmentation methods. Phys. Med 2015;31:969-80.

[39] Gui L, Li C, Yang X. Medical image segmentation based on level set and isoperimetric constraint. Phys Med 2017;42:162-73. 